\title{
Tropopause-Level Waveguides
}

\author{
O. MARTIUS \\ Institute for Atmospheric and Climate Science, ETH Zurich, Zurich, Switzerland \\ C. SCHWIERZ \\ Seminar for Statistics, ETH Zurich, Zurich, Switzerland \\ H. C. DAVIES \\ Institute for Atmospheric and Climate Science, ETH Zurich, Zurich, Switzerland
}

(Manuscript received 29 October 2008, in final form 28 August 2009)

\begin{abstract}
Jet streams located on the dynamical tropopause are accompanied by coaligned bands of enhanced potential vorticity (PV) gradients, and these bands can serve as space-time evolving waveguides for synoptic and larger-scale flow. Consideration is given to the detection and examination of the along-flow extent and lateral confinement of the waveguides on the dynamic tropopause (i.e., iso-PV surface) and on tropopause-cutting isentropic surfaces. Thereafter a two-part study is undertaken of the possible interaction between such waveguides (i.e., jet streams). First, a highly idealized theoretical model points to the nature of the dynamical linkage that can exist between perturbations on coaligned waveguides. Second, diagnostic analysis of two observed events helps identify the contemporaneous space-time evolution of the jets and serves to illustrate the nature of the transfer of wave activity from the extratropical waveguide onto a subtropical and a polar waveguide, respectively.
\end{abstract}

\section{Introduction}

Tropopause-level jet streams are accompanied by a steplike decrease in the height of the tropopause itself, and there is an accompanying strong lateral gradient of the potential vorticity (PV) across the step. Also, on isentropic surfaces that traverse the step from the troposphere to the stratosphere, this PV gradient, $\sim 10 \mathrm{PV}$ units (PVU; where $1 \mathrm{PVU}=1 \times$ $10^{-6} \mathrm{~m}^{2} \mathrm{~s}^{-1} \mathrm{~K} \mathrm{~kg}^{-1}$ ) in $1000 \mathrm{~km}$ (Davies and Rossa 1998; Schwierz et al. 2004), takes the form of a narrow and elongated band aligned along the jet. A major dynamical repercussion is that adiabatic displacement of air across the band will induce significant PV perturbations. For balanced flow the resulting PV perturbations will significantly influence the in situ flow and could in turn both instigate perturbations on un-

Corresponding author address: Olivia Martius, Institute for Atmospheric and Climate Science, ETH Zurich, Zurich 8092, Switzerland.

E-mail: olivia@env.ethz.ch derlying surface fronts and/or result in propagation of the perturbations along the jet.

The existence of this narrow band of enhanced PV gradient has prompted theoretical consideration of the flow dynamics associated with an ambient zonally aligned PV discontinuity. It has been shown that the discontinuity can sustain both small-amplitude Rossby-like waves trapped to and propagating along the discontinuity (Platzman 1968) and stationary large-amplitude meanders of the jet itself (Swanson et al. 1997). It has further been shown that such waves can be triggered and maintained by a nearby localized PV anomaly (Schwierz et al. 2004).

Also, recognizing the propensity for waves to propagate along the meandering jet stream, Martius et al. (2006) refined the conventional Hovmöller diagram to track the progression of realized waves along the jet itself. In effect, their approach entails examining the longitude-time trace of the meridional velocity on the jet, and it thereby circumvents the latitudinal averaging process that accompanies the conventional Hovmöller approach. In the foregoing studies the jet is in effect viewed as a laterally confined Rossby waveguide that 


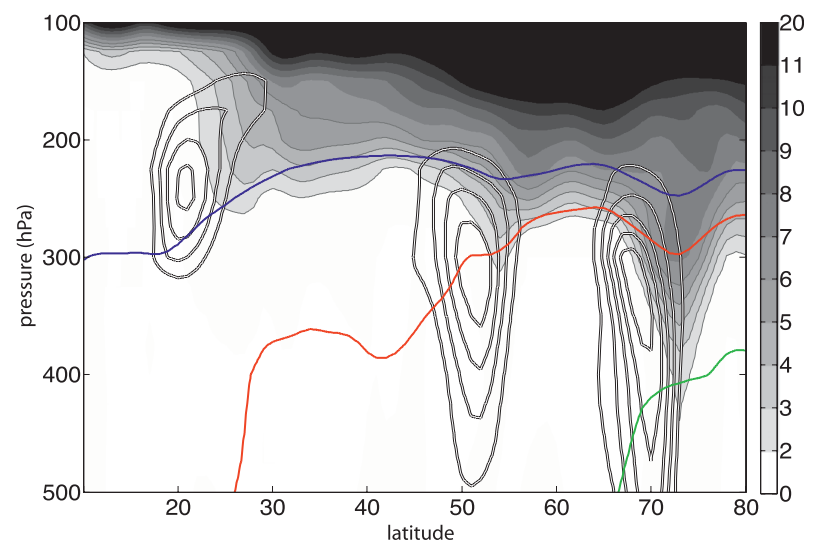

FIG. 1. An instantaneous "latitude-pressure" cross section along $15^{\circ} \mathrm{W}$ on 1200 UTC 20 Jan 2001 . Shown are the PV pattern (shaded, PVU), the wind velocity (black and white contours) between 30 and $50 \mathrm{~m} \mathrm{~s}^{-1}$ at $5 \mathrm{~m} \mathrm{~s}^{-1}$ increments, and the location of three isentropes $(300 \mathrm{~K}$, green; $320 \mathrm{~K}$, red; $340 \mathrm{~K}$, blue). Aspects of the contemporaneous patterns on quasi-horizontal surfaces are shown in Fig. 2.

evolves in space-time and is conducive to the quasilongitudinal transmission of wave packets. This perspective is also adopted in the present study.

This study is itself motivated by the contemporaneous occurrence of multiple jet streams in the same longitudinal band. The frequency of such occurrences has been adduced (Koch 2004; Koch et al. 2006) by compiling a climatology based on the 15-yr European Centre for Medium-Range Weather Forecasts (ECMWF) Re-Analysis (ERA-15) dataset of both single and double jet events. It was shown that during the Northern Hemisphere winter a double jet configuration is more prevalent than a single jet within the longitudinal band $40^{\circ} \mathrm{W}-40^{\circ} \mathrm{E}$, and the subtropical jet over North Africa has an accompanying jet located poleward of it for more than $50 \%$ of the time. The equivalent in the Southern Hemisphere winter is the longitudinal band $\left(80^{\circ}-320^{\circ} \mathrm{E}\right)$ and the elongated jet region extending from far west of central Australia to far east of the South Island of New Zealand.

For illustrative purposes and subsequent reference, a triple jet structure is shown in Fig. 1a [cf. the schematic of Shapiro and Keyser (1990) in their Fig. 10.5]. It features the co-occurrence of a subtropical jet, an extratropical (polar) jet, and possibly an Arctic jet, and the accompanying topography of the tropopause (the 2-PVU isoline) resembles a series of irregular steps. Moreover, each step is associated with a strong lateral PV gradient, and the overall configuration can be viewed as a localized and instantaneous occurrence of the more familiar diffuse climatologically averaged "PV staircase" (Dritschel and McIntyre 2008).
The dynamics and geometric constraints governing the appearance of multiple jet streams in the time-mean field of planetary and geophysical flow systems has been examined in numerous studies (e.g., Lee and Kim 2003; Williams 2003). In contrast, the focus here is on the dynamics associated with the day-to-day co-occurrence and evolution of multiple atmospheric waveguides (i.e., jet streams). The regular coexistence of multiple jets basis prompts questions related to their interdependence and possible interaction.

The remainder of the paper is structured as follows. In section 2, a diagnostic framework is set out both for identifying waveguides and for examining the contemporaneous evolution and linkage of perturbations on spatially separate waveguides. In section 3 , a conceptual understanding is sought on this coevolution by formulating and analyzing the dynamics of a highly idealized theoretical model. Thereafter, in section 4 two case studies are presented to illustrate the coevolution of separate waveguides, and finally in section 5 an overview is given of the results, together with some further general comments on the significance of such flow developments.

\section{Diagnostic framework}

\section{a. Analysis fields}

The case study analyses undertaken in this study are conducted using the 40-yr ECMWF Re-Analaysis (ERA-40) dataset (Uppala et al. 2005). The primary atmospheric fields from this set are interpolated onto a $1^{\circ} \times 1^{\circ}$ geographical grid, and thereafter two-dimensional patterns are derived of the following fields: a scaled measure of the gradient of the potential vorticity on tropopause-intersecting isentropes, the height of the dynamical tropopause (taken as the 2-PVU isosurface), and the analyzed and geostrophic wind field plus the gradient of the potential temperature field on the tropopause. These derived fields will form the basis for identifying contemporaneous multiple waveguides (jets) and for diagnosing their evolution and interaction.

\section{b. Diagnostic approach}

There are two mutually related goals to our diagnosis. The first is to identify and assess the dynamical character and evolution of the jet as encapsulated by its accompanying band of enhanced PV gradient on tropopausetraversing isentropes. One measure of the band is $\nabla_{\theta}(\mathrm{IPV})$, where IPV is the isentropic, hydrostatic form of the Ertel potential vorticity.

Here we adopt a variant of this measure, namely $\nabla_{\theta}[\ln (\mathrm{IPV})]$. This selection is prompted by noting the asymmetry in the amplitude of the flow response attributable to significant $\pm \Delta \mathrm{PV}$ anomalies of the isentropic 
potential vorticity (IPV) away from the ambient state. In effect, the response to positive (negative) anomalies is substantially weaker (stronger). In contrast, the flow response attributable to local $\pm \Delta q$ anomalies of the quasigeostrophic potential vorticity $(q)$ would equate to opposite but equal velocity perturbations.

Here we set out the rationale for the selection of the $\nabla_{\theta}[\ln (\mathrm{IPV})]$ variant. Isentropic potential vorticity takes the form

$$
\mathrm{IPV}=\frac{(\zeta+f)}{P},
$$

with $\zeta$ and $f$ denoting respectively the vertical components of the relative and earth vorticity on an isentropic surface and $P=-(1 / g) \partial p / \partial \theta$.

On partitioning both $f$ and $P$ into background values, $f_{0}$ and $P_{0}=P_{0}(\theta)$, and deviation components $\left(f^{\prime}\right.$ and $\left.P^{\prime}\right)$ that is, $f=\left(f_{0}+f^{\prime}\right)$ and $P=\left(P_{0}+P^{\prime}\right)$-it follows that

$$
\ln (\mathrm{IPV})=\ln \left\{f_{0}\left[1+\frac{\left(\zeta+f^{\prime}\right)}{f_{0}}\right]\right\}-\ln \left\{P_{0}\left[1+\frac{P^{\prime}}{P_{0}}\right]\right\} .
$$

Thus, to first order,

$$
\ln (\mathrm{IPV}) \approx \ln \left(\frac{f_{0}}{P_{0}}\right)+\frac{1}{f_{0}} q,
$$

with $q=\left[\zeta+f^{\prime}-\left(f_{0} / P_{0}\right) P^{\prime}\right]$. Hence the IPV gradient, $\nabla_{\theta}(\mathrm{IPV})$, is linked to the quasigeostrophic gradient, $\left(\nabla_{\theta} q\right)$, by the relationship

$$
\nabla_{\theta}[\ln (\mathrm{IPV})] \approx \frac{1}{f_{0}} \nabla_{\theta} q .
$$

In effect, $f_{0} \nabla_{\theta}[\ln (\mathrm{IPV})]$ is akin to a quasigeostrophic measure for the atmosphere's potential vorticity gradient (i.e., the atmosphere's "effective" $\beta$ ), and its adoption facilitates the interpretation of the waveguide dynamics within a quasigeostrophic framework. Note that the logarithmic expansion employed above is only useful if $\left|P^{\prime}\right| \ll|P(\theta)|$ on a $\theta$ surface, and this is only marginally valid. Nonetheless, adoption of this pseudoquasigeostrophic variant provides

(i) a simplified setting for theoretical considerations (cf. Nielsen-Gammon and Gold 2008);

(ii) a first-order quasigeostrophic assessment of the relative strength of the PV gradients associated with the contemporaneous jets, while de-emphasizing finer-scale PV structures in the stratosphere that are not directly related to waveguide dynamics; and

(iii) a concise relationship between the jet flow $(U)$ and the quasigeostrophic gradient (cf. Davies 1981),

$$
\nabla_{\theta}^{2} U=-\nabla_{\theta} q .
$$

Our second diagnostic objective is to determine the nature of the interaction of perturbations on the two waveguides. A seemingly attractive single-level analysis would be to examine the flow evolution on the dynamic tropopause since (a) the prevailing jets tend to reside on this surface and are identifiable as narrow meandering streams of fast-flowing air, (b) the jets are usually collocated with bands of enhanced gradients both of tropopause height (i.e., $\nabla_{\text {trop }} H$ ) and of potential temperature (i.e., $\nabla_{\text {trop }} \theta$ ), and (c) potential temperature is a quasiconserved variable on this isosurface and its bands of enhanced gradient might serve as a proxy to depict the attendant waveguide dynamics.

The foregoing factors prompt and underpin the socalled " $\theta$ on a PV surface" portrayal of tropopause-level flow. However, for our present purposes it has an intrinsic shortcoming, and this can be illustrated as follows. The expression for the geostrophic velocity $\left(\mathbf{v}_{G}\right)$ on an iso-PV surface (dynamic tropopause) is

$$
f \mathbf{v}_{G}=\mathbf{k} \times\left[\nabla_{\mathrm{PV}} M-\left(c_{p} T\right) \nabla_{\mathrm{PV}}(\ln \theta)\right],
$$

where $M=\left(c_{p} T+g z\right)$ denotes the Montgomery function. On the 2-PVU isosurface the two terms on the right-hand side substantially counter one another. The $\nabla_{\mathrm{PV}} M$ term usually connotes a supergeostrophic contribution to the flow at the jet's location, and the $\nabla_{\mathrm{PV}}(\ln \theta)$ term is usually of comparable value but opposite in sign. In effect, the $\nabla_{\mathrm{PV}}(\ln \theta)$ pattern on the dynamic tropopause neither represents the jet location nor captures the attendant Rossby waveguide, and perturbations need not necessarily propagate along such a band. This limits the use of the " $\theta$ on a PV surface" perspective in isolation to examine the dynamics of the waveguides.

Note that no such cancellation prevails on an isentropic surface where

$$
f \mathbf{v}_{G}=\mathbf{k} \times \nabla_{\theta} M
$$

and the $M$ and $\theta$ surfaces are aligned at a much more acute angle.

\section{c. Illustrative example}

The import of the above considerations is illustrated in Fig. 2. The panels provide a sectorial display of the wind strength on the tropopause (Fig. 2a), the strength of the geostrophic flow $\left[\nabla_{\mathrm{PV}} M-\left(c_{p} T\right) \nabla_{\mathrm{PV}}(\ln \theta)\right] / f$ (Fig. 2b), the patterns of $\nabla_{\theta}[\ln (\mathrm{IPV})]$ on the $320-\mathrm{K}$ and the $340-\mathrm{K}$ isentropic surfaces for $\mathrm{PV} \geq 1$ (Figs. $2 \mathrm{c}, \mathrm{d}$ ), and the tropopause topography (Fig. 2e) and the $\left(\nabla_{\text {trop }} \theta\right)$ gradient on the tropopause (Fig. 2f). 

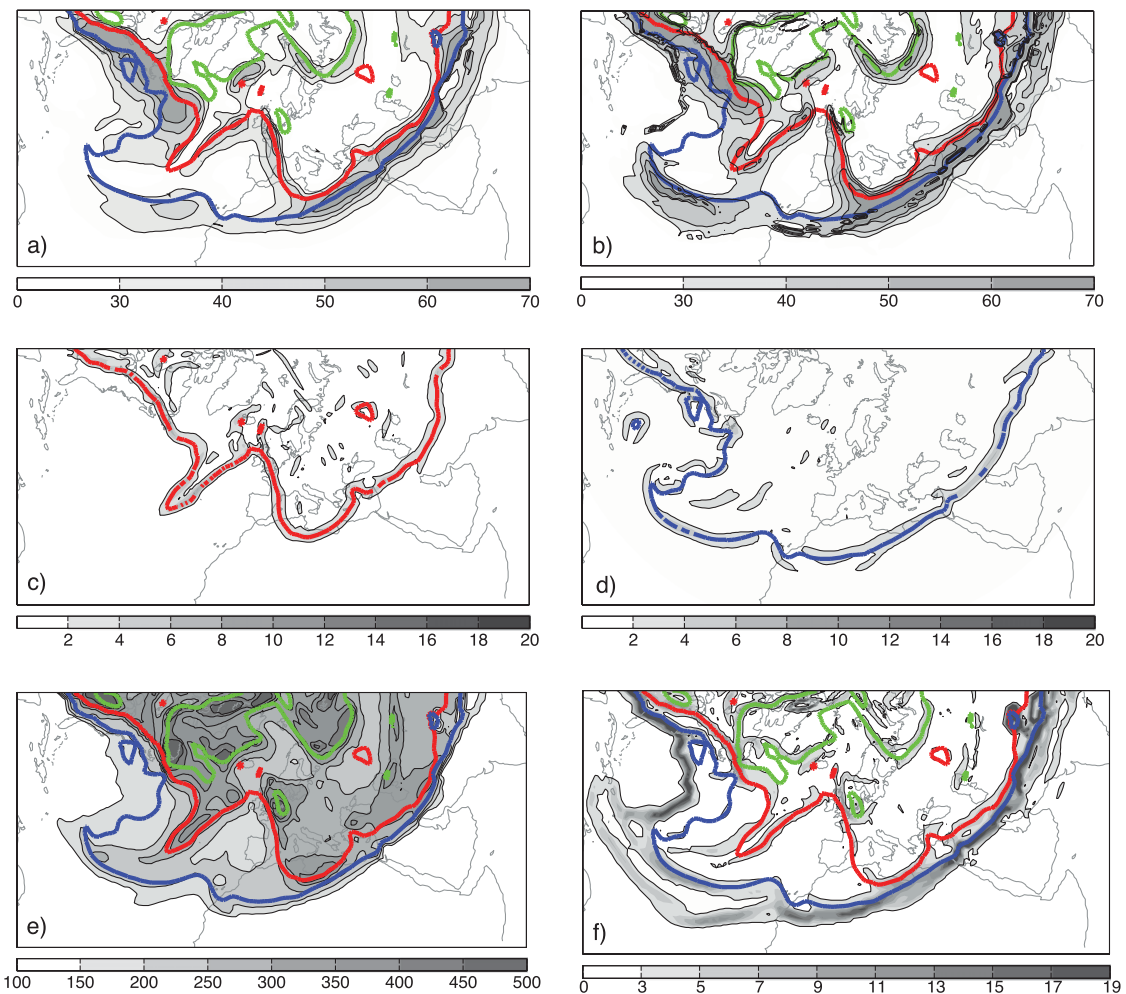

FIG. 2. Depictions of the flow in the Atlantic-European sector on 1200 UTC 20 Jan 2001. The panels depict (a) wind strength (shaded, $\mathrm{m} \mathrm{s}^{-1}$ ) on the dynamical tropopause and the contours representing the intersection of the dynamical tropopause with the 300 (green), 320 (red), and $340 \mathrm{~K}$ (blue) potential temperature isosurfaces; (b) the geostrophic wind field $\left[\nabla_{\mathrm{PV}} M-\right.$ $\left.\left(c_{p} T\right) \nabla_{\mathrm{PV}}(\ln \theta)\right] / f\left(\mathrm{~m} \mathrm{~s}^{-1}\right)$ on the dynamical tropopause; (c),(d) the $\ln (\mathrm{PV})$ gradient on the (c) 320- and (d) 340-K isentropic surfaces for PV > 1 PVU, together with the location of the 2 PVU isoline $\left(1000 \mathrm{~km}^{-1}\right)$; and (e),(f) the height of the dynamical tropopause (shaded) in pressure and the gradient of the potential temperature $\left(10^{-5} \mathrm{~km}^{-1}\right)$ on the dynamical tropopause (shaded) plus the intersection of the dynamical tropopause with the 300- (green), 320- (red), and $340-\mathrm{K}$ (blue) isentropes.

The flow configuration is fairly complex, but Fig. 2a suggests the coexistence of subtropical, extratropical, and Arctic jets (cf. also Fig. 1b). Further inspection of this panel indicates that over the western North Atlantic the jets coalesce to form a single deep vertically aligned jet stream, whereas over the eastern Atlantic they acquire separate identities, and further downstream in the Eastern Hemisphere the extratropical and subtropical jets merge. The transient vertical superposition of jets in some geographical sectors has already been noted (cf. Shapiro et al. 1999, including their Fig. 1) and is a frequent feature over the eastern Pacific (Riehl 1962). Figure $2 b$ shows that the geostrophic flow bears a reasonable correspondence to the realized flow field.

The $\nabla_{\theta}[\ln (\mathrm{IPV})]$ patterns on the 320 - and $340-\mathrm{K}$ surfaces (Figs. 2c,d) each capture a single spatially coherent and laterally highly confined waveguide that bear comparison with the extratropical and subtropical jets, respectively. These figures also support the identification of the
2-PVU isoline as a proxy for the location of the jet and the waveguide. Noting that the parameter $f_{0} \nabla \theta[\ln ($ IPV) $]$ corresponds the "effective $\beta$ " of the flow, these two panels indicate that the extratropical and subtropical waveguides are of amplitude $2-8 \times 10^{-10} \mathrm{~m}^{-1} \mathrm{~s}^{-1}$ and hence are respectively significantly more than one order of magnitude larger than the ambient field and the in situ value of $\beta$.

Figure 2e demonstrates, in line with the earlier comments, that the jets are collocated with sharp changes in the tropopause topography, yielding at different longitudes one, two, or three steplike features. Figure $2 \mathrm{f}$ confirms that the $\left(\nabla_{\operatorname{trop}} \theta\right)$ pattern has a rich spatial structure and replicates to a measure the meanders of the three jets but, in harmony with the earlier caveat, also carries some elongated signatures (e.g., over the subtropical Atlantic) that do not coincide with the presence of a jet.

From the above we conclude that the $\nabla_{\theta}[\ln ($ IPV) $]$ patterns on differing isosurfaces capture the spatial location 
and strength of the waveguide, whereas the $\left(\nabla_{\text {trop }} \theta\right)$ pattern allied with the depiction of key isentropes has the advantage of roughly capturing the jet locations on a single surface.

\section{Theoretical considerations}

The recognition that tropopause-level jet streams can constitute elongated bands of $\nabla_{\theta}[\ln ($ IPV $)]$ that are highly confined laterally and whose amplitude is at least an order of magnitude larger than the ambient gradient has important dynamical ramifications. It implies that the free atmosphere's Rossby wave dynamics is closely allied to, and indeed can be dominated by, these waveguide bands. Further, it invites viewing the atmosphere's large and synoptic-scale flow dynamics as being highly influenced by two factors: first, the interaction of the bands with one another and their surface equivalent (the elongated and narrow frontal bands of enhanced baroclinicity) and second, the influence on a band of isolated PV anomalies located in its neighborhood.

From this perspective the interaction of a tropopauselevel band with a surface front corresponds to the setting for baroclinic development, albeit in a richly structured basic flow. Likewise, the influence of a tropopause PV anomaly with a surface frontal band corresponds to the classical upper-level triggering of surface cyclogenesis, and such an anomaly can also instigate a wave train on a contiguous tropopause-level band (Schwierz et al. 2004).

Here the focus is on the hitherto substantially unexplored theme of the interaction of perturbations on two tropopause-level waveguides. To this end we examine the nature of the dynamics of a highly idealized but germane flow setting, and in the same context comment on the more general implications of the aforementioned waveguide perspective.

\section{a. An idealized model setting}

Here a conceptual understanding is sought on the nature of the interaction between two tropopause-level waveguides. We consider the simple flow setting of barotropic flow on a midlatitude $\beta$ plane and then examine the dynamics of small-amplitude perturbations of a basic state comprising two zonally aligned jets. The basic state (see Fig. 3 for a schematic depiction) is prescribed by three zonally aligned bands of uniform absolute vorticity $\left(q_{1}, q_{2}, q_{3}\right)$ separated by PV discontinuities of strength $\Delta_{I}\left(=q_{1}-q_{2}\right)$ and $\Delta_{I I}\left(=q_{2}-q_{3}\right)$ located at $y= \pm a$. This configuration is selected such that both $\Delta_{I}$ and $\Delta_{I I}>0$, and this rules out the occurrence of barotropic instability. The associated zonal flow takes the form
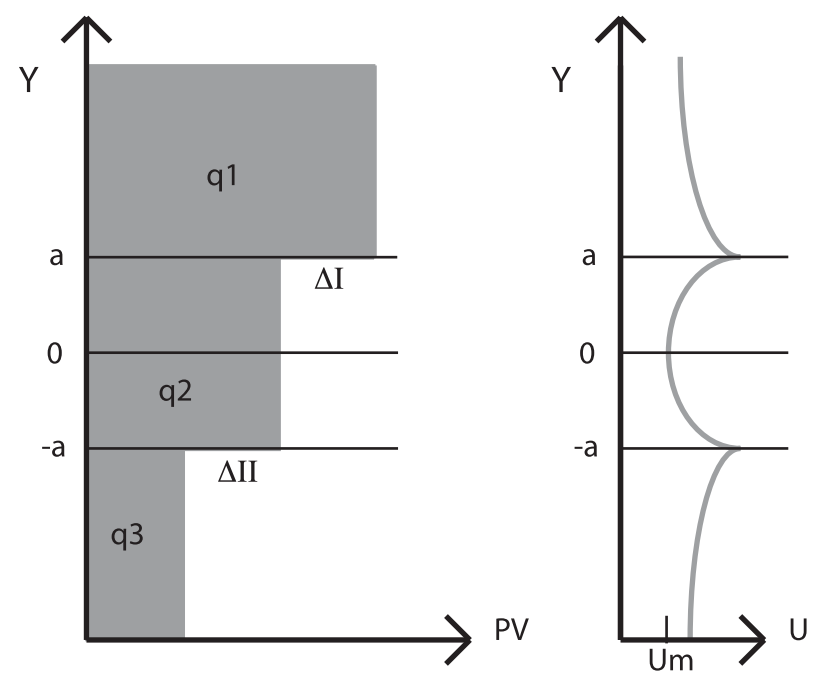

FIG. 3. A schematic depiction of a three-layer-two-step PV distribution and the associated basic state zonal velocity field.

$$
\begin{array}{llrl}
U=U_{m}-q_{1}(y-a)-q_{2} a+\frac{1}{2} \beta y^{2} & \text { for } & y>a, \\
U=U_{m}-q_{2} y+\frac{1}{2} \beta y^{2} & \text { for } & -a<y<a, \\
U=U_{m}-q_{3}(y+a)+q_{2} a+\frac{1}{2} \beta y^{2} & \text { for } & y<-a,
\end{array}
$$

so that the two jets are collocated with the vorticity discontinuities at $y= \pm a$, and the velocity $U_{m}$ at $y=0$ is a prescribed constant. Note that this is an overtly idealized model; later we will comment on the model's limitations.

\section{b. Dynamics of the perturbed flow}

Small-amplitude perturbations of this basic state are governed by the linearized barotropic vorticity equation,

$$
\left(\frac{\partial}{\partial t}+U \frac{\partial}{\partial x}\right) \zeta^{\prime}+v^{\prime} \frac{\partial Q}{\partial y}=0
$$

where $Q$ denotes the basic state absolute vorticity, and $\left(\psi^{\prime}, v^{\prime}, \zeta^{\prime}\right)$ represent respectively the perturbation streamfunction, meridional velocity, and vorticity, so that $v^{\prime}=$ $\partial \psi^{\prime} / \partial x$ and $\zeta^{\prime}=\nabla^{2} \psi^{\prime}$.

For wavelike disturbances, the perturbation vorticity in each of the three bands is zero, and waves can only occur across the PV steps. Hence the present simple model configuration is designed to focus on the nature and strength of the interaction between the waves that can exist on the respective waveguides. The perturbed streamfunction can be written in the following compact two-component form: 


$$
\psi^{\prime}=\psi_{I}^{\prime}+\psi_{I I}^{\prime}
$$

with

$$
\begin{array}{ll}
\psi_{I}^{\prime}=A e^{-k(y-a)} \cos \left(k x+\varepsilon_{I}\right) & y>a, \\
\psi_{I}^{\prime}=A e^{+k(y-a)} \cos \left(k x+\varepsilon_{I}\right) & y<a,
\end{array}
$$

and

$$
\begin{array}{ll}
\psi_{I I}^{\prime}=B e^{-k(y+a)} \cos \left(k x+\varepsilon_{I I}\right) & y>-a, \\
\psi_{I I}^{\prime}=B e^{+k(y+a)} \cos \left(k x+\varepsilon_{I I}\right) & y<-a .
\end{array}
$$

Here $A$ and $B$ indicate the time-dependent wave amplitudes, $k$ is the wavenumber, and $\varepsilon$ the wave phase. In effect, the perturbation components $\psi_{I}^{\prime}$ and $\psi_{I I}^{\prime}$ correspond respectively to waves trapped on the jets located respectively at $y= \pm a$. A key feature of this formulation is that the velocity induced by a perturbation on one waveguide (located at, say, $y=a$ ) has a nonzero value at the location of the second waveguide (at $y=-a$ ) and hence can influence the latter's evolution.

Integrating Eq. (3.2) across the discontinuity at $y=a$ from $[a-l, a+l]$ in the limit of $l \rightarrow 0$ yields the relationship

$$
\left(\frac{\partial}{\partial t}+U \frac{\partial}{\partial x}\right)\left[\left.\psi_{y}^{\prime}\right|_{a+}-\left.\psi_{y}^{\prime}\right|_{a-}\right]+\left.\Delta_{I} v^{\prime}\right|_{a}=0 .
$$

Insertion of the expressions for $\psi^{\prime}$ from Eqs. (3.3) into the above relationship, and in the analog relationship at $y=-a$, yields the following linked time-dependent equations for the coevolution of amplitudes $[A(t), B(t)]$ of the two trapped waves and their relative phase $(\delta=$ $\left.\varepsilon_{I}-\varepsilon_{I I}\right)$ :

$$
\begin{aligned}
\frac{\partial A}{\partial t} & =\frac{1}{2}\left(\gamma \Delta_{I}\right) B \sin \delta \\
\frac{\partial B}{\partial t} & =-\frac{1}{2}\left(\gamma \Delta_{I I}\right) A \sin \delta \\
\frac{\partial \delta}{\partial t} & =-P+\frac{1}{2} \gamma\left[\Delta_{I}\left(\frac{B}{A}\right)-\Delta_{I I}\left(\frac{A}{B}\right)\right] \cos \delta
\end{aligned}
$$

with

$$
\begin{aligned}
P & =\left[k\left(U_{a}-U_{-a}\right)-\frac{1}{2}\left(\Delta_{I}-\Delta_{I I}\right)\right] \\
& =\left[-2 k a q_{2}-\frac{1}{2}\left(\Delta_{I}-\Delta_{I I}\right)\right], \text { and } \gamma=e^{-2 k a} .
\end{aligned}
$$

This triplet of equations [Eqs. $(3.4 \mathrm{a}-\mathrm{c})]$ is analogous to those derived for the classical Eady baroclinic setting (Davies and Bishop 1994) and the barotropic instability problem (Heifetz et al. 2004).

The triplet prescribes the nature of the interaction between the waves on the two waveguides. Equations $(3.4 a, b)$ indicate that the wave on one waveguide modifies the amplitude of the wave on the other guide and that the strength of the influence depends on (i) the spatial separation of the jets $(2 a)$ and the wavelength $(k)$ of the perturbations (via $\gamma$, a geometric factor), (ii) the strength of the two vorticity discontinuities $\left(\Delta_{I}, \Delta_{I I}\right)$, and (iii) the relative phase $\delta$ of the two waves such that if $\psi_{I}$ leads $\psi_{I I}$ (i.e., $\pi<\delta<2 \pi$ ), then the amplitude $B$ increases and $A$ decreases with the reverse applying for $\psi_{I I}$ leading $\psi_{I}$ (i.e., $0<\delta<\pi$ ).

An interpretation of Eq. (3.4c) follows from noting that the phase velocities $\left(V_{1}, V_{2}\right)$ of the $\psi_{I}^{\prime}$ and $\psi_{I I}^{\prime}$ waves propagating in isolation of one another are given by $\left[\left(U_{a}-1 / 2 \Delta_{I} / k\right),\left(U_{-a}-1 / 2 \Delta_{I I} / k\right)\right]$ (see Swanson et al. 1997), so that $\left(k^{-1} P\right)=V_{1}-V_{2}$. Hence, Eq. (3.4c) indicates that the time rate of change of the relative phase $(\delta)$ depends on the difference between $P$ and the strength of the influence of the perturbation on the other waveguide. Note also that $P=0$ if the phase velocities $\left(V_{1}, V_{2}\right)$ are equal. This prevails when there is an exact compensation between the relative effect on the phase speeds of the stronger (weaker) vorticity discontinuity and the stronger (weaker) ambient flow velocity.

It is pertinent to make two further remarks regarding the triplet of equations. First, assigning a positive or negative value to the vorticity $\left(q_{2}\right)$ of the middle layer introduces an asymmetry into the basic state, but there is no a priori justification for the choice. For the derivation detailed in the next subsection (and the appendix), we set $q_{2}=0$.

Second, the studies by Davies and Bishop (1994) and Heifetz et al. (2004) considered an unstable basic state and the dynamics correspond to the interaction of counterpropagating edge or Rossby waves as they transit toward a phase-locked state. In contrast, the basic state considered herein is stable, and the interacting waves are not counterpropagating and their relative phase changes continuously.

\section{c. Temporal invariants and a special solution}

In the appendix it is shown that the triplet of Eqs. (3.4) possess two temporal invariants,

$$
\begin{gathered}
\left(\Delta_{I I} A^{2}+\Delta_{I I} B^{2}\right)=\text { constant and } \\
\left\{A^{2}+B^{2}-\left[\left(\Delta_{I}-\Delta_{I I}\right) \frac{\gamma}{P}\right] A B \cos \delta\right\}=\text { constant. }
\end{gathered}
$$


The temporal invariant of Eq. (3.5a) is the limit for the present model of the more general barotropic area integral invariant:

$$
\frac{\delta}{\delta t} \iint_{S}\left[\left(\frac{\delta q}{\delta y}\right)^{-1} \frac{1}{2} \zeta^{\prime 2}\right] d s=0 .
$$

In effect, Eq. (3.5a) is related to the conservation of pseudomomentum, and the invariant connotes the transfer of pseudomomentum from one waveguide to the other. Hereafter we shall refer to this transfer loosely as an "energy exchange."

The two invariants place constraints on the evolution of the flow. Thus, if the initial state comprises of only a perturbation $A_{0}$ on the waveguide at $y=a$ (i.e., $\left.A\right|_{t=0}=A_{0}$ with $\left.B\right|_{t=0}=B_{0}=0$ ), it follows from Eqs. (3.5a) that

$$
B^{2}=\left(\frac{\Delta_{I I}}{\Delta_{I}}\right)\left(A_{0}^{2}-A^{2}\right)
$$

This leaves open the possibility that the amplitude $B$ of the $\psi_{I I}^{\prime}$ wave might attain a large value provided $\left(\Delta_{I I} / \Delta_{I}\right) \gg$ 1. For the specified initial state, Eq. (3.5b) reduces to

$$
\cos \delta=-\Phi\left(\frac{B}{A}\right), \text { with } \Phi=\frac{\left[1-\left(\frac{\Delta_{I}}{\Delta_{I I}}\right)\right]}{2 \gamma} .
$$

It follows that $\delta=3 \pi / 2$ initially and the $B$ perturbation grows. The growth rate decreases as the waves move out of quadrature with $\delta$ decreasing toward $\pi$, and the growth of the $B$ wave ceases and reverses when $\delta$ passes through this value. The rate of growth is small and the rate of phase change large if $\gamma\left(=e^{-2 k a}\right)$ is small.

For the aforementioned initial flow setting (i.e., $\left.A\right|_{t=0}=$ $A_{0}$ with $\left.B\right|_{t=0}=B_{0}=0$ ), the evolution of the amplitude $B$ of $\psi_{I I}^{\prime}$ wave takes the form (see the appendix)

$$
\frac{B^{2}}{A_{0}^{2}}=\left(\frac{1}{\chi^{2}}\right) \sin ^{2}(\chi p t)
$$

with $p=(1 / 2)\left(\gamma \Delta_{I I}\right)$ and $\chi^{2}=\left(\Delta_{I} / \Delta_{I I}\right)+\left[P^{2} /\left(\gamma \Delta_{I I}\right)^{2}\right]$. In effect, the waves undergo a vacillation with a period $\pi /(\chi p)$, and the $\psi_{I I}^{\prime}$ wave attains its peak amplitude, $B_{\max }=(1 / \chi) A_{0}$, after a time $\pi /(2 \chi p)$. It follows that a significant amplitude enhancement, $B / A_{0}$, is achievable only after an extended time period, and this militates against rapid growth.

To illustrate this, consider two limit forms of Eq. (3.7). First if $P=0$, so that $\chi^{2}=\left(\Delta_{I} / \Delta_{I I}\right)$, the maximum achievable amplitude, $B_{\max }=\left(\Delta_{I I} / \Delta_{I}\right)^{1 / 2} A_{0}$, is realized. This occurs for a basic state with $\Delta_{I I}=\Delta_{I}$, i.e., with $q_{1}=-q_{3}$.
In this circumstance the time $T^{*}$ required to attain this maximum amplitude is

$$
T^{*}=\frac{\pi}{\gamma \sqrt{\Delta_{I} \Delta_{I I}}} .
$$

Thus, for a specified basic state, there is a perturbation wavelength, $k=k^{*}$, that will allow a small-amplitude wave on a weak waveguide to induce a larger-amplitude wave on a strong waveguide, but the accompanying time scale $T^{*}$ will be large if $\gamma \ll 1$.

For the second limit, assume $P$ is large, such that $\chi \approx$ $P /\left(\gamma \Delta_{I I}\right)$. It follows that for this setting,

$$
B_{\max } \approx\left(\frac{\gamma \Delta_{I I}}{P}\right) A_{0}, \quad \text { and } \quad T^{*}=\frac{\pi}{P}
$$

This corresponds to only modest amplitude enhancement $\left(B_{\max } \leq \sqrt{2} A_{0}\right)$ occurring on synoptic time scales ( $T^{*} \sim 2.5$ days) for a wide range of $2 \mathrm{ka}$ values.

\section{d. Remarks}

The idealized theoretical model considered above was adopted to provide a conceptual handhold on the dynamics that can prevail when two tropopause-level waveguides (jet streams) exist in isolation and are embedded in an environment of zero background PV gradient. For this setting it was shown that the influence of a wave perturbation trapped on one waveguide could instigate perturbations on the other waveguide. This influence is strongly dependent on the spatial separation of the waveguides and on the relative phase of the two wave perturbations (strongest when the waves are in quadrature; i.e., $\delta=\pi / 2$ or $3 \pi / 2$ ). If the relative strength of the two waveguides is large, then the foregoing effect can result in a significant amplitude enhancement.

Further general inferences for this simple setting are twofold. First, the transient but substantial interwave exchange militates against the sustained existence of two waveguides in a comparatively undisturbed state if they are initially coaligned in close proximity. Second, the interwave exchange is conducive to a growing wave approaching more closely the other waveguide at some longitude and/or the growing wave breaking to form a cutoff as it becomes subject to the deformation effects of the large-scale flow. In either case, the flow development is then subject to nonlinear effects, and in the case of a cutoff it could in turn trigger or sustain waves on the other waveguide.

Thus the model, although overtly oversimplified, does shed light on the nature of the possible interaction. In the final section we comment further on the model's limitations and point to possible refinements. Here we 


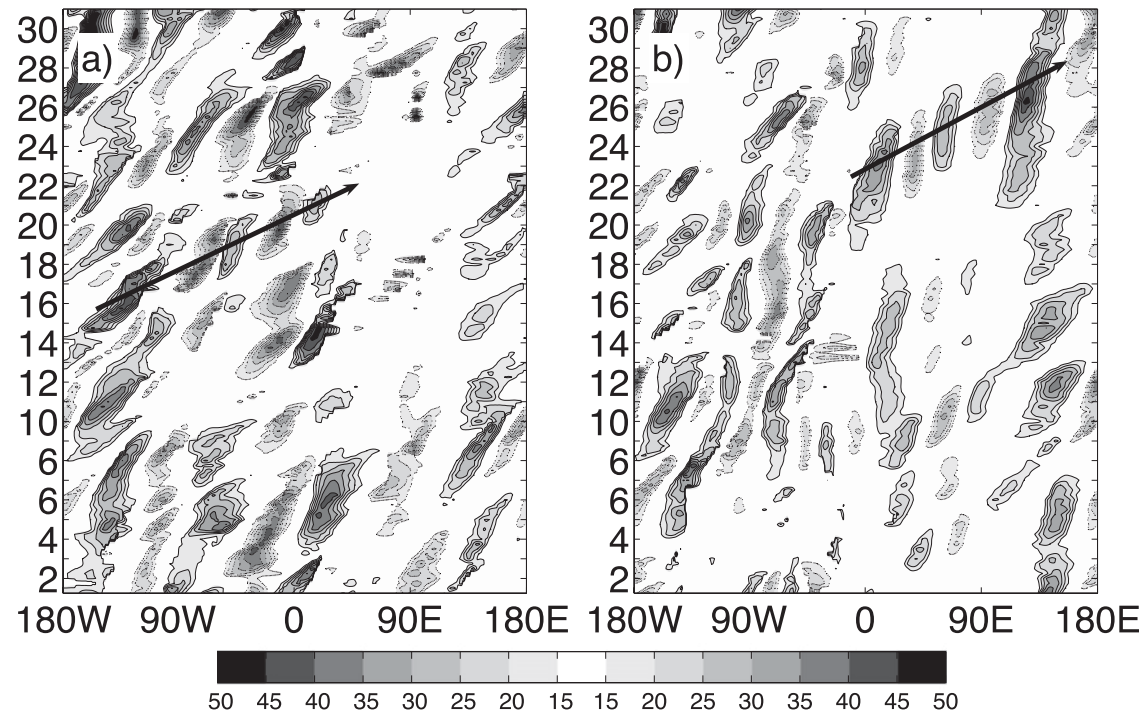

FIG. 4. Hovmöller diagrams of the meridional wind velocity $\left(\mathrm{m} \mathrm{s}^{-1}\right)$ along the dynamical tropopause (see text for further details) in November 1991 for the (a) 320- and (b) 340-K isentropic surface. The arrows indicate the wave trains mentioned in the text.

proceed to examine realized examples of synoptic flow evolution within the framework of jet interaction portrayed by this simple model.

\section{Examples of jet-jet interaction}

In this section we provide consecutive examples of equatorward transfer of wave energy from the extratropical (polar) to the subtropical waveguide and of poleward transfer from the extratropical to the Arctic waveguide.

\section{a. Equatorward transfer}

Figure 4 shows refined Hovmöller diagrams (see Martius et al. 2006) of the meridional velocity on two different isentropic levels for the month of November 1991. Refined Hovmöller diagrams show longitude-time slices of an atmospheric variable (here the meridional velocity) averaged on an isentropic surface across a narrow meridional band centered on the dynamical tropopause (2-PVU isoline). For the present purpose, note that in wintertime the extratropical dynamical tropopause intersects the $320-\mathrm{K}$ isosurface and the subtropical tropopause intersects the $340-\mathrm{K}$ surface. On the $320-\mathrm{K}$ surface a wave train is seen to peter out over Europe (at approximately $20^{\circ} \mathrm{E}$ ) around 20 November (Fig. 4a). Concurrently on the $340-\mathrm{K}$ surface a wave train emerges and progresses eastward away from Europe (again initially appearing near $20^{\circ} \mathrm{E}$ ) (Fig. $4 \mathrm{~b}$ ). Leading up to this transition there is a hint of a transient alignment of the wave trains on the two waveguides with a quarter of a wavelength phase shift between $90^{\circ} \mathrm{W}$ and $0^{\circ}$. This is in harmony with the interpretation provided in the previous section of the coupled dynamics of waveguide perturbations.

To study the synoptic character of the development during this phase between 18 and 22 November, we show a sequence of depictions of the wind strength (Fig. 5) and the $\nabla_{\theta}[\ln (\mathrm{IPV})]$ patterns on the $320-$ and $340-\mathrm{K}$ surfaces (Fig. 6). At an early phase of this transition period (0000 UTC 18 November) a trough is located around $60^{\circ} \mathrm{W}$ (label A in Figs. 5 and $6 \mathrm{a}$ ). West of $40^{\circ} \mathrm{W}$ the subtropical and the extratropical waveguides are in spatial proximity. The main wave signal propagates along the extratropical waveguide. It is baroclinic in structure and the wave signal extends down to the surface (not shown). The isentropic PV gradient $\nabla_{\theta}[\ln ($ IPV) $]$ allied to the $320-\mathrm{K}$ isentrope is enhanced over an extended longitudinal range west of $30^{\circ} \mathrm{E}$. At this time there is no evidence of significant wave activity along the $340-\mathrm{K}$ isentrope over Asia, and the subtropical waveguide is weak in comparison (Fig. 6b).

Two days later (0600 UTC 20 November), a significant ridge has developed over the eastern Atlantic with a downstream trough near the Greenwich meridian. At this time there is an indication of a transfer from the extratropical onto the subtropical waveguide (label B in Fig. 5). A relatively coherent band of enhanced $\nabla_{\theta}[\ln (\mathrm{IPV})]$ is evident on both 320 - and $340-\mathrm{K}$ surfaces east of the zero meridian (Fig. 6c,d).

A day later (0600 UTC 21 November), the trough over Europe acquires a northeast-southwest tilt (see the 

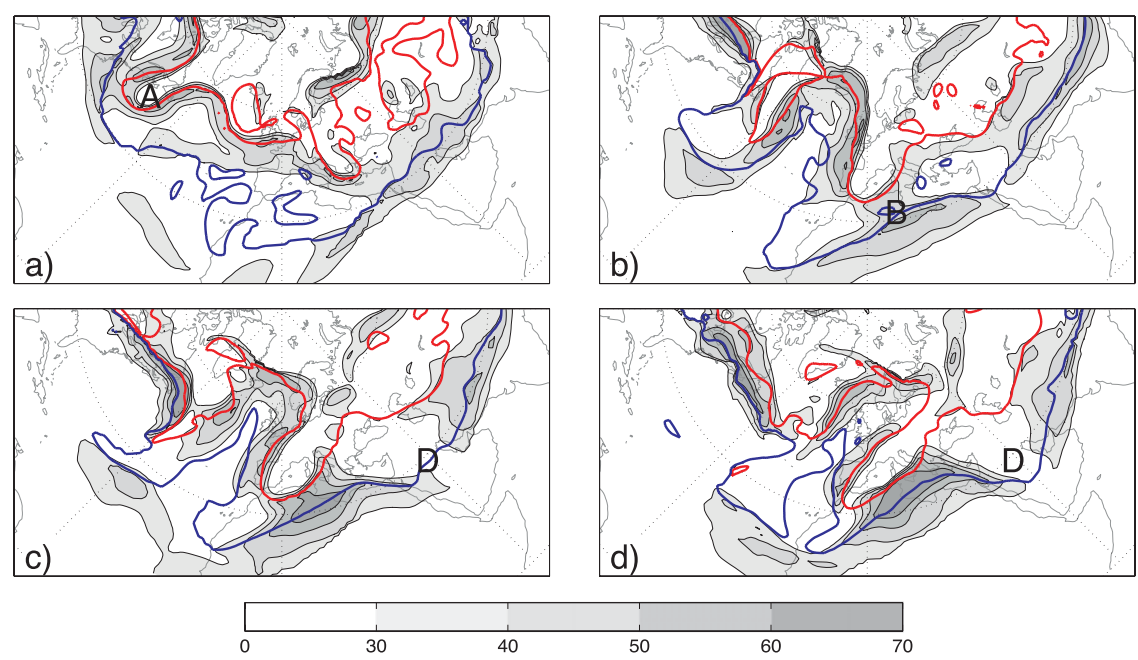

FIG. 5. Wind field on the dynamical tropopause (shaded, $\mathrm{m} \mathrm{s}^{-1}$ ). Also shown are the contours of the intersection of the dynamical tropopause with the 320- (red) and 340-K (blue) potential temperature surfaces at (a) 0000 UTC 18 Nov, (b) 0600 UTC 20 Nov, (c) 0600 UTC 21 Nov, and (d) 0600 UTC 22 Nov 1991.

feature labeled C in Fig. 6e), and the energy transfer onto the subtropical waveguide has progressed with evidence of a downstream development along this waveguide (label D in Fig. 5c) with an accompanying (Figs. 6e,f) coherent and undulating band of enhanced $\nabla_{\theta}[\ln (\mathrm{IPV})]$ present along the 340-K contour, stretching from Africa all through Asia. The wave signal on the subtropical waveguide is confined to upper levels (450-150 hPa) and it does not exhibit a westward tilt with height (not shown). In this context, note that the low-level baroclinicity beneath the subtropical jet over Africa tends to be weak (Koch et al. 2006), thereby inhibiting the occurrence of deep baroclinic development on the jet at this longitude.

A further $24 \mathrm{~h}$ later (0600 UTC 22 November), the main wave signature is on the subtropical waveguide (label D in Fig. 5e), and the wave signal extends to about $80^{\circ} \mathrm{E}$. The streamer over Europe is further elongated. Two days later (0000 UTC 24 November; not shown) the wave train on the subtropical waveguide is fully developed, the streamer over Europe has cut off, and there is no evidence of wave energy on the extratropical guide over Europe and Asia.

\section{b. Poleward transfer}

An example of poleward energy transfer from the extratropical to the Arctic waveguide occurred in January 2000. The event is depicted in Figs. 7 and 8 that constitute the counterpart of Figs. 5 and 6 for this case.

The initial situation (0000 UTC 28 January) shows a strong and coherent extratropical and waveguide in the western Atlantic (Figs. 8a,b). It overlays the Arctic waveguide at around $50^{\circ} \mathrm{W}$ (cf. Figs. $7 \mathrm{a}, 8 \mathrm{a}, \mathrm{b}$ ), but they bifurcate again at around $35^{\circ} \mathrm{W}$ (see labels $\mathrm{A}$ and $\mathrm{B}$ in Figs. $7 \mathrm{a}$ and $8 \mathrm{a}, \mathrm{b})$. Other notable features include a breaking wave with a major ridge over the eastern Atlantic and an elongated trough that extends from western Europe equatorward into the Atlantic.

One day later (0000 UTC 29 January) there is an indication of the initiation of wave activity on the Arctic waveguide between $20^{\circ} \mathrm{W}$ and $0^{\circ}$ (see label C in Figs. $7 \mathrm{~b}$ and $8 \mathrm{c}, \mathrm{d})$. Downstream of the breaking wave over Europe the extratropical jet merges with the subtropical jet south of the Mediterranean and over Asia (not shown), and a wave train appears on this feature. The strength and coherence of the Arctic waveguide is more clearly evident in the $\nabla_{\theta}[\ln ($ IPV) $]$ field (Figs. $8 \mathrm{c}, \mathrm{d}$ ).

Another day later (0000 UTC 30 January) the extratropical and Arctic waveguides are in phase west of the Greenwich meridian but bifurcate east of the zero meridian. An incipient wave train that appears on the Arctic waveguide and extends eastward (Figs. 7c,d and $8 \mathrm{e}, \mathrm{f}$ ) is clearly separated from the subtropical jet in the same longitudinal band. This downstream wave train is located to the north of the main baroclinic zone and confined in the vertical to the middle to upper troposphere.

The analyses of these two observational cases, in line with the results from the idealized model, point to a transfer of perturbation energy from the extratropical jet onto another waveguide. Again, in line with the model results, the first case study provides evidence that integral to the transfer is the presence of waves on the 

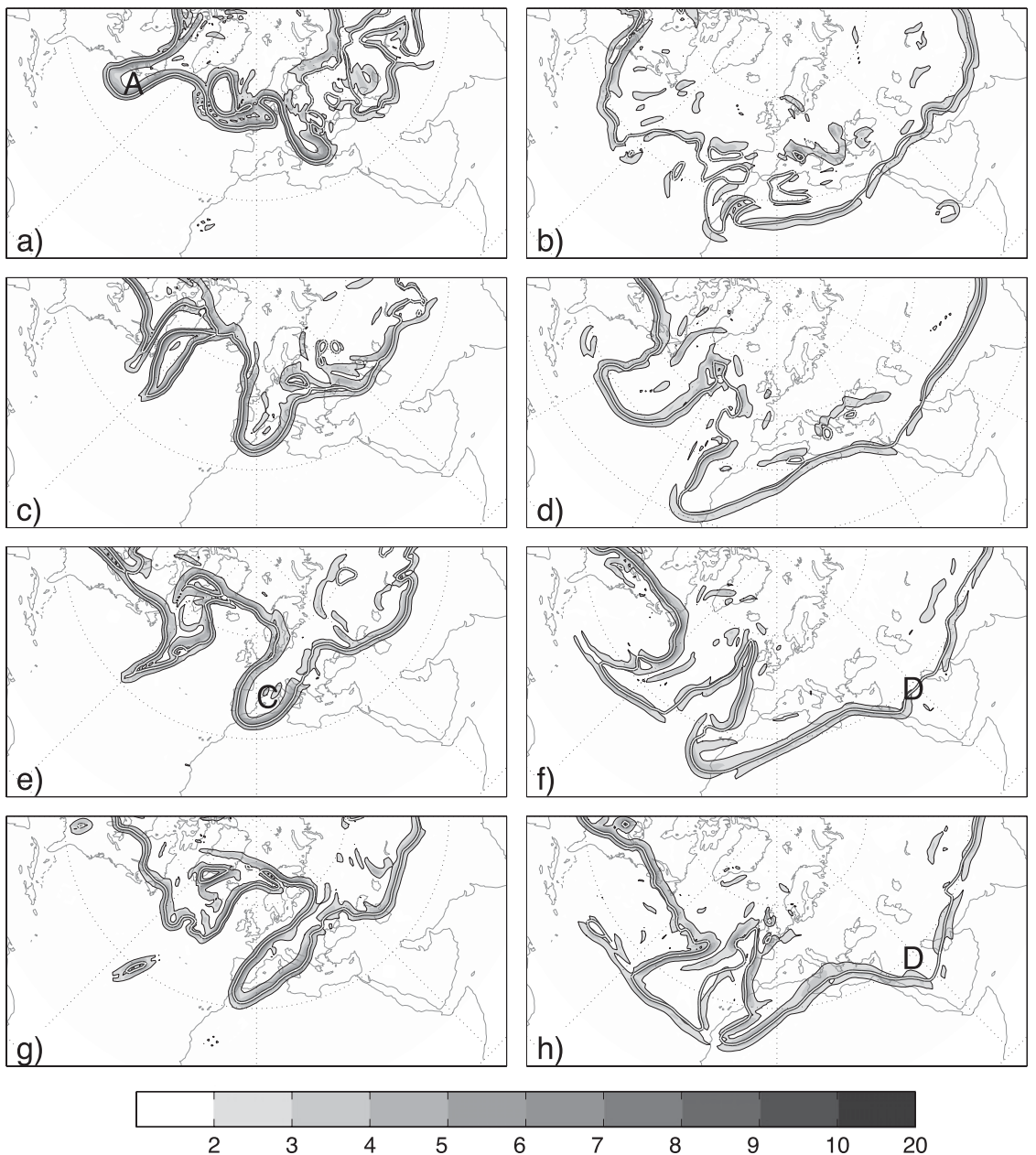

FIG. 6. The $\ln (\mathrm{PV})$ gradient for PV $>1$ PVU on the (left) 320- and (right) 340-K isentropic surfaces (shaded, $1000 \mathrm{~km}^{-1}$ ) with 2-PVU contour (solid line) for (a),(b) 0000 UTC 18 Nov, (c),(d) 0600 UTC 20 Nov, (e),(f) 0600 UTC 21 Nov, and (g),(h) 0600 UTC 22 Nov 1991.

two wave guides with a quadrature phase shift. However, a striking feature of the realized cases was the occurrence ahead of the transfer of a strong meridional extension of the extratropical jet and a possible breaking of the dilated wave to form a cutoff flow feature. Such a pronounced meridional extension often occurs during the final phase of a baroclinic development, and hence the process is influenced by low-level and upper-level interaction. The meridional extension is clearly conducive to the transfer of perturbation energy.

Indeed, for the case of transfer to the subtropical jet, the extratropical wave breaks and the attendant PV distribution can concomitantly strengthen the adjacent subtropical waveguide (see Figs. 6g,h). For the case of transfer to the pre-existing strong and coherent Arctic jet, the latter undercuts the extratropical jet and thereby contributes to the strong cyclonic shear poleward of the jet.

\section{Further remarks}

Central to the present study was the recognition that tropopause-level jets are bands of enhanced PV gradient on isentropic surfaces that serve as waveguides for perturbations, and that double jet stream structures are a regular feature of the atmospheric flow. In this study a combination of diagnostic considerations, a simple theoretical model and case study analyses have been employed to shed light on the dynamics of double jet streams. Here we comment critically on these three aspects.

First, the diagnostic considerations point to the value of the amalgam of the PV perspective that identifies the waveguide bands of enhanced $\nabla_{\theta}[\ln (\mathrm{IPV})]$ and the $\theta$ perspective that captures ingredients of the coevolution of the jets on a single surface.

Second, the simple theoretical model points to the dynamics of the interaction of double jet streams and 

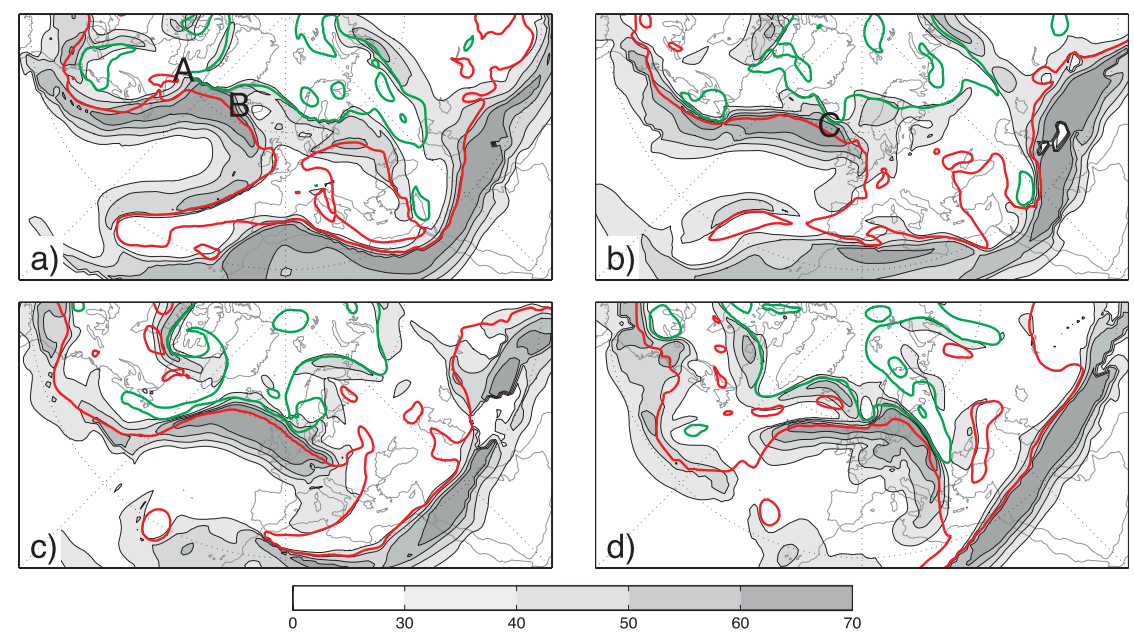

FIG. 7. Wind strength (shaded, $\mathrm{m} \mathrm{s}^{-1}$ ) on the dynamical tropopause. Contours indicate the intersection of the dynamical tropopause with the 300- (green) and 320-K (red) isentropic surfaces for (a) 0000 UTC 28 Jan, (b) 0000 UTC 29 Jan, (c) 0000 UTC 30 Jan, and (d) 0000 UTC 31 January 2000.

the possibility of a limited transfer of pseudomomentum/ perturbation energy between the waveguides (jets). It was noted earlier that the basic state adopted in the present study is not unstable [cf. Eqs. $(3.4 \mathrm{a}, \mathrm{b})$ if $\Delta_{I}$ and $\Delta_{I I}$ were opposite in sign]. In effect, the resulting flow vacillation is an example of pseudomomentum/perturbation energy exchange in a stable setting. Such an exchange also prevails for the classical Orr mechanism, but the dynamics is fundamentally different. In its simplest barotropic form, the Orr mechanism can arise from the geometric rearrangement of perturbation vorticity by a uniform basic state shear flow possessing a zero vorticity gradient. In effect, the Orr mechanism requires the prescription (generation) of vorticity perturbations within a uniform vorticity flow. In contrast, for our double waveguide setting the vorticity of the perturbed flow is generated dynamically at the vorticity discontinuities of the basic state.

The theoretical model is overtly oversimplified. Possible refinements could include limiting the width of the flow domain to exclude the unrealistic zonal velocity of the basic state at large lateral distances and introducing weak ambient vorticity gradients between the waveguides and nondiscontinuous vorticity jumps. To a first order it is to be expected that these adjustments would merely introduce weak quantitative differences. It would also be of interest to consider wave packet effects as opposed to a single wavelength and allow for the nonalignment of the guides and their finite downstream extent. The present analysis constitutes a foundation for studies of this type.

Likewise, it would be desirable to extend the model to incorporate a range of additional atmospheric-like fac- tors, in particular three-dimensional effects including the coupled dynamics of tropopause-level waveguides and the surface baroclinic waveguide beneath the extratropical jet. These effects include baroclinic development and instability and are of first order. Here the approach has been to examine tropopause-level waveguide dynamics in isolation while acknowledging the prevalence and strength of the interlevel coupling.

Third, the two observational case studies revealed complex flow evolutions that included the merging and bifurcation of waveguides (jets) while the aforementioned jet-to-jet transfer of pseudomomentum-perturbation energy was accompanied by strongly nonlinear effects associated with wave breaking. A related issue is the climatological significance of the jet-to-jet transfer of pseudo-momentum-perturbation energy. A climatological regression analysis of the band-passed streamfunction field during winter over eastern Europe (Lee 2000) gives a hint that the transition of waves from the extratropical to the subtropical jet is a climatologically robust phenomenon, and likewise that the transfer can occur in either direction (see Fig. 3 from Lee 2000). A climatological analysis of wave precursors to winter precipitation in Israel hints at the relevance of the interwaveguide wave transfer for these specific weather events (Feldstein and Dayan 2008).

Finally, the co-occurrence of double waveguides emphasized in this study underlines the need for sensitive and refined diagnostic approaches to tease out the nature of the accompanying dynamics and concomitantly highlights the complexity of realized along-jet propagation of perturbation energy and interjet energy transfer. 

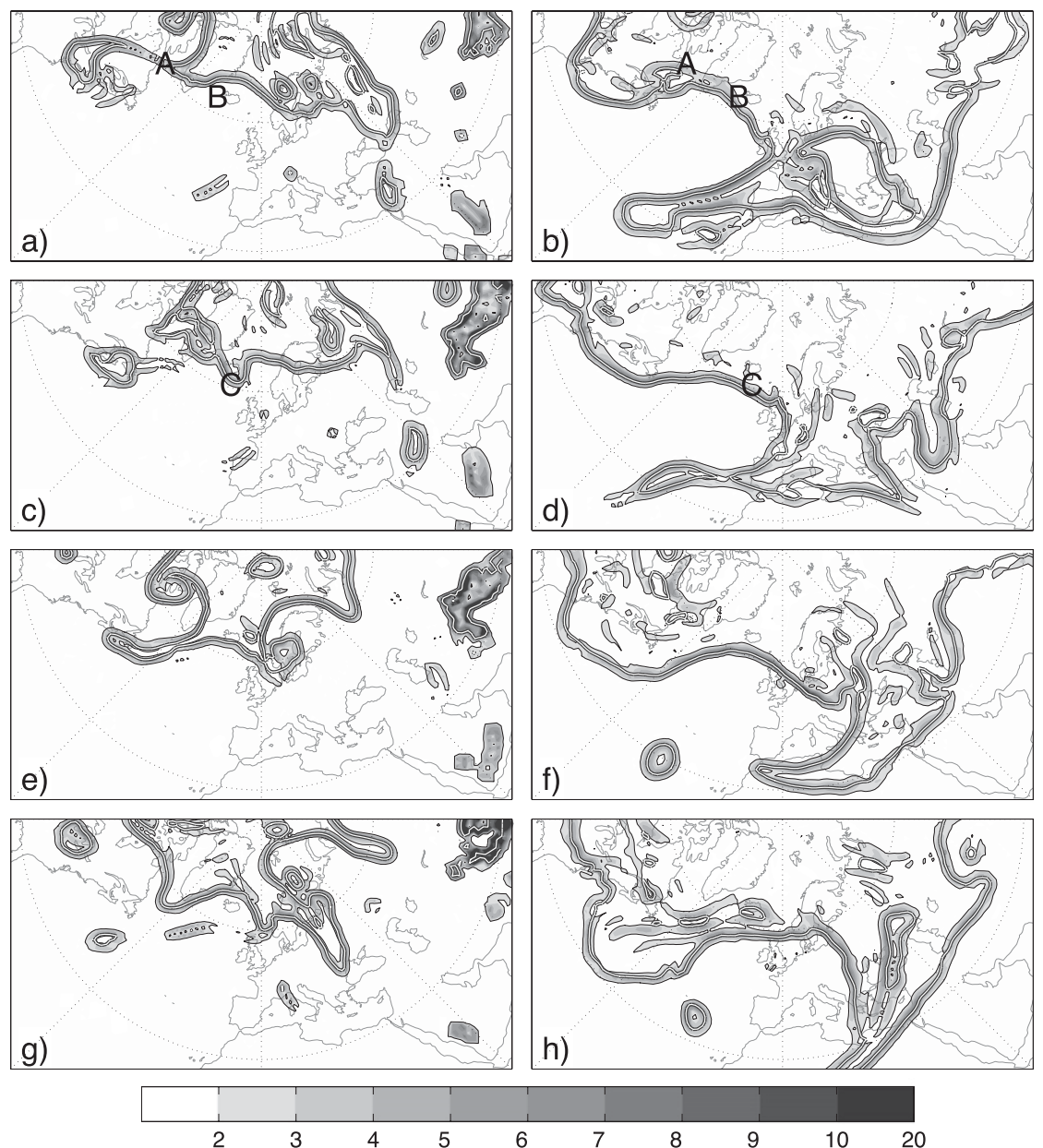

FIG. 8. The $\ln (\mathrm{PV})$ gradient for PV $>1 \mathrm{PVU}$ (shaded) $\left(1000 \mathrm{~km}^{-1}\right)$ on the (left) $300-$ and (right) 320- isentropic surfaces together with the 2-PVU contour (solid line) for (a),(b) 0000 UTC 28 Jan, (c),(d) 0000 UTC 29 Jan, (e),(f) 0000 UTC 30 Jan, and (g),(h) 0000 UTC 31 Jan 2000.

Acknowledgments. The authors thank MeteoSwiss for providing access to the ERA-40 dataset. We gratefully acknowledge the support of the NCCR-Climate Programme of the SNF in funding the research and express our appreciation to Mike Blackburn and three anonymous reviewers for many helpful comments.

\section{APPENDIX}

\section{Derivation of Invariants and a Specific Solution}

Here we derive two invariants of the system of Eqs. (3.4) and also the solution set out in Eq. (3.7).

\section{a. Temporal invariants}

Multiplying Eq. (3.4a) by $\Delta_{I I} A$ and Eq. (3.4b) by $\Delta_{I} B$ and adding the resulting equations immediately delivers the temporal invariant:

$$
\frac{\partial\left(\Delta_{I I} A^{2}+\Delta_{I} B^{2}\right)}{\partial t}=0 .
$$

Note also that Eqs. (3.4a) and (3.4b) can be combined to show that

$$
\frac{\partial\left(A^{2}+B^{2}\right)}{\partial t}=\gamma A B\left(\Delta_{I}-\Delta_{I I}\right) \sin \delta .
$$

To derive the second invariant, we first multiply Eq. (3.4a) by $B$ and Eq. (3.4b) by $A$, and then add the resulting equations to yield

$$
\frac{\partial A B}{\partial t}=\frac{1}{2} \gamma\left(\Delta_{I} B^{2}-\Delta_{I I} A^{2}\right) \sin \delta .
$$

Likewise, Eq. (3.4c) can be rearranged into the form 


$$
A B \frac{\partial \delta}{\partial t}=-P(A B)+\frac{1}{2} \gamma\left(\Delta_{I} B^{2}-\Delta_{I I} A^{2}\right) \cos \delta .
$$

Eliminating the term $\left[1 / 2(\gamma)\left(\Delta_{I} B^{2}-\Delta_{I I} A^{2}\right)\right]$ from the last two equations yields successively

$$
\begin{aligned}
A B \sin \delta \frac{\partial \delta}{\partial t}-\cos \delta \frac{\partial(A B)}{\partial t} & =-P(A B) \sin \delta \quad \text { and } \\
\frac{\partial(A B \cos \delta)}{\partial t} & =+P(A B) \sin \delta .
\end{aligned}
$$

Finally, substituting the expression for $[(A B) \sin \delta]$ from Eq. (A.2) into Eq. (A.3) delivers the second invariant:

$$
\frac{\partial\left\{\left(A^{2}+B^{2}\right)-\left[\frac{\left(\Delta_{I}-\Delta_{I I}\right) \gamma}{P}\right] A B \cos \delta\right\}}{\partial t}=0 .
$$

\section{b. Specific solution}

For an initial state comprising only a perturbation $A_{0}$ on the waveguide at $y=a$ (i.e., $\left.A\right|_{t=0}=A_{0}$ with $\left.B\right|_{t=0}=0$ ), it follows from Eqs. (A.1) and (A.4) respectively that

$$
B^{2}=\left(\frac{\Delta_{I I}}{\Delta_{I}}\right)\left(A_{0}^{2}-A^{2}\right)
$$

and

$$
\cos \delta=-\Phi\left(\frac{B}{A}\right)
$$

with

$$
\Phi=\frac{\left[1-\left(\frac{\Delta_{I}}{\Delta_{I I}}\right)\right]}{2 \gamma} .
$$

On substituting the expression for $B$ from (A.5) and the expression for sin $\delta$ derivable from (A.6) into the equation for the evolution for the amplitude tendency [i.e., Eq. (3.4b)], that is,

$$
\frac{\partial B}{\partial t}=-\frac{1}{2}\left(\gamma \Delta_{I I}\right) A \sin \delta
$$

it follows that

$$
\frac{\partial B}{\partial t}=-p \chi\left[\left(\frac{A_{0}}{\chi}\right)^{2}-B^{2}\right]^{1 / 2}
$$

with

$$
p=\frac{1}{2}\left(\gamma \Delta_{I I}\right) \quad \text { and } \quad \chi^{2}=\left\{\frac{\Delta_{I}}{\Delta_{I I}}+\frac{P^{2}}{\left(\gamma \Delta_{I I}\right)^{2}}\right\} .
$$

Thus, $(p \chi) d t=d B /\left[\left(A_{0} / \chi\right)^{2}-B^{2}\right]^{-1 / 2}$ and integrating from $t=0$ to $t=T$ yields

$$
(p \chi) T=\left[\Psi_{T}-\Psi_{0}\right]
$$

where

$$
\sin \Psi=\frac{B}{\left(\frac{A_{0}}{\chi}\right)}
$$

so that

$$
\cos (p \chi T)=\left[1-\frac{B^{2}}{\left(A_{0} / \chi\right)^{2}}\right]^{1 / 2}
$$

and hence we have the solution set out in Eq. (3.7):

$$
\frac{B^{2}}{A_{0}^{2}}=\left(\frac{1}{\chi^{2}}\right)\left[\sin ^{2}(\chi p t)\right] .
$$

\section{REFERENCES}

Davies, H. C., 1981: An interpretation of sudden warmings in terms of potential vorticity. J. Atmos. Sci., 38, 427-445.

— velopment. J. Atmos. Sci., 51, 1930-1946.

—_, and A. M. Rossa, 1998: PV frontogenesis and uppertropospheric fronts. Mon. Wea. Rev., 126, 1528-1539.

Dritschel, D. G., and M. E. McIntyre, 2008: Multiple jets as PV staircases: The Philips effect and the resilience of eddytransport barriers. J. Atmos. Sci., 65, 855-874.

Feldstein, S. B., and U. Dayan, 2008: Circumglobal teleconnections and wave packets associated with Israeli winter precipitation. Quart. J. Roy. Meteor. Soc., 134, 455-467.

Heifetz, E., C. H. Bishop, B. J. Hoskins, and J. Methven, 2004: The counter-propagating Rossby-wave perspective on baroclinic instability. I: Mathematical basis. Quart. J. Roy. Meteor. Soc., 130, 211-231.

Koch, P., 2004: Novel perspectives of jet-stream climatologies and events of heavy precipitation on the alpine southside. ETH Zurich Rep. 15622, 203 pp.

— , H. Wernli, and H. C. Davies, 2006: An event-based jet-stream climatology and typology. Int. J. Climatol., 26, 283-301.

Lee, S., 2000: Barotropic effects on atmospheric storm tracks. J. Atmos. Sci., 57, 1420-1435.

—, and H.-K. Kim, 2003: The dynamical relationship between subtropical and eddy-driven jets. J. Atmos. Sci., 60, 14901503.

Martius, O., C. Schwierz, and H. C. Davies, 2006: A refined Hovmöller diagram. Tellus, 58A, 221-226.

Nielsen-Gammon, J. W., and D. A. Gold, 2008: Potential vorticity diagnosis of the severe convective regime. Part II: The impact of idealized PV anomalies. Mon. Wea. Rev., 136, 1582-1592. 
Platzman, G. W., 1968: The Rossby wave. Quart. J. Roy. Meteor. Soc., 94, 225-248.

Riehl, H., 1962: Jet streams of the atmosphere. Tech. Paper 32, Department of Atmospheric Science, Colorado State University, $117 \mathrm{pp}$.

Schwierz, C., S. Dirren, and H. C. Davies, 2004: Forced waves on a zonally aligned jet stream. J. Atmos. Sci., 61, 73-87.

Shapiro, M. A., and D. Keyser, 1990: Fronts, jet streams and the tropopause. Extratropical Cyclones: The Eric Palmén Memorial Volume, C. W. Newton and E. O. Holopainen, Eds., Amer. Meteor. Soc., 167-191.
— - and Coauthors, 1999: A planetary-scale to mesoscale perspective on the life cycles of extratropical cyclones: The bridge between theory and observations. The Life Cycles of Extratropical Cyclones, M. A. Shapiro and S. Grønås, Eds., Amer. Meteor. Soc., 139-185.

Swanson, K. L., P. J. Kushner, and I. M. Held, 1997: Dynamics of barotropic storm tracks. J. Atmos. Sci., 54, 791-810.

Uppala, S. M., and Coauthors, 2005: The ERA-40 Re-Analysis. Quart. J. Roy. Meteor. Soc., 131, 2961-3012.

Williams, G. P., 2003: Jovian dynamics. Part III: Multiple, migrating, and equatorial jets. J. Atmos. Sci., 60, 1270-1296. 